\title{
PLGA-based control release of Noggin blocks the premature fusion of cranial sutures caused by retinoic acid
}

\author{
Weicai Wang ${ }^{1} \cdot$ Chen Zhou $^{1} \cdot$ Zhicai Feng $^{1} \cdot$ Hongyu $\mathrm{Li}^{1} \cdot$ Yadong Zhang $^{1} \cdot$ Baicheng Bao $^{1} \cdot$ Bin Cai $^{1} \cdot$ Mu Chen ${ }^{2}$. \\ Hongzhang Huang ${ }^{1}$ (D)
}

Received: 31 July 2018 / Revised: 29 September 2018 / Accepted: 9 October 2018 / Published online: 3 November 2018

(C) The Author(s) 2018

\begin{abstract}
Craniosynostosis (CS), the premature and pathological fusion of cranial sutures, is a relatively common developmental disorder. Elucidation of the pathways involved and thus therapeutically targeting it would be promising for the prevention of CS. In the present study, we examined the role of BMP pathway in the all-trans retinoic acid (atRA)-induced CS model and tried to target the pathway in vivo via PLGA-based control release. As expected, the posterior frontal suture was found to fuse prematurely in the atRA subcutaneous injection mouse model. Further mechanism study revealed that atRA could repress the proliferation while promote the osteogenic differentiation of suture-derived mesenchymal cells (SMCs). Moreover, BMP signal pathway was found to be activated by atRA, as seen from increased expression of BMPR-2 and pSMAD1/5/9. Recombinant mouse Noggin blocked the atRA-induced enhancement of osteogenesis of SMCs in vitro. In vivo, PLGA microsphere encapsulated with Noggin significantly prevented the atRA-induced suture fusion. Collectively, these data support the hypothesis that BMP signaling is involved in retinoic acid-induced premature fusion of cranial sutures, while PLGA microsphere-based control release of Noggin emerges as a promising strategy for prevention of atRA-induced suture fusion.
\end{abstract}

Keywords Craniosynostosis $\cdot$ PLGA microsphere $\cdot$ BMP $\cdot$ Noggin

\section{Introduction}

Craniosynostosis (CS), the premature fusion of one or more of the cranial sutures, which causes secondary deformations of the cranial vault, cranial base, and brain, occurs with an estimated birth prevalence of 1 in 2000 to 2500 live births

Weicai Wang and Chen Zhou contributed equally to this work.

$\mathrm{Mu}$ Chen

chenmove@163.com

Hongzhang Huang

hhzhang@mail.sysu.edu.cn

Weicai Wang

wangwc3@mail.sysu.edu.cn

Chen Zhou

zhouch46@mail.sysu.edu.cn

Zhicai Feng

fengzhc@mail.sysu.edu.cn

Hongyu Li

lihy65@mail.sysu.edu.cn worldwide (Johnson and Wilkie 2011; Wilkie et al. 2010). Craniosynostosis can lead to dramatic clinical manifestations in terms of cosmesis and functional impairment including craniofacial deformation, mental retardation, and difficulties with vision, hearing, and breathing (Wilkie and Morriss-Kay 2001). Approximately $21 \%$ of craniosynostosis patients have

\author{
Yadong Zhang \\ zhangyad@mail2.sysu.edu.cn \\ Baicheng Bao \\ baobaicheng@163.com \\ Bin Cai \\ caib@mail.sysu.edu.cn \\ 1 Guanghua School of Stomatology, Hospital of Stomatology, \\ Guangdong Provincial Key Laboratory of Stomatology, Sun Yat-sen \\ University, 56 Lingyuanxi Road, Guangzhou, China \\ 2 Department of Stomatology, Shenzhen Nanshan People's Hospital \\ and The 6th Affiliated Hospital of Shenzhen University Health \\ Science Center, 89 Taoyuan Road, Shenzhen, China
}


pathologic genetic alterations ( $86 \%$ single-gene mutations and $14 \%$ chromosomal abnormalities) (Wilkie et al. 2010). Craniosynostosis has also been associated with environmental factors including fetal constraint and maternal tobacco, alcohol, and drug use (Carmichael et al. 2008; Gardner et al. 1998; Honein and Rasmussen 2000; Jentink et al. 2010; Kallen 1999; Olshan and Faustman 1989; Sanchez-Lara et al. 2010; Zeiger et al. 2002). Despite the recent progress in molecular biology and using animal models, many details of the suture biology and the pathophysiological mechanisms of craniosynostosis remain unknown.

Retinoic acid (RA), a metabolite of vitamin A, plays a key role in a variety of biological processes and is essential for normal embryonic development (Rhinn and Dolle 2012). However, retinoic acid is also a notorious teratogenic factor causing craniofacial deformations including craniosynostosis (Gardner et al. 1998). High doses of retinoic acid on prenatal Macaca nemestrina result in the premature formation of all coronal sutures (Yip et al. 1980). Microarray analysis showed that the level of retinol-binding protein 4 (RBP4), a transport protein for retinol in serum, was decreased 37-fold in prematurely fused/fusing sutures compared to unfused sutures (Coussens et al. 2007). RBP4 was also found to be significantly downregulated during mineralization of human cranial suture mesenchyme cells, analogous to its decrease in pathological suture fusion (Leitch et al. 2013). Moreover, human null and hypomorphic mutations in the gene encoding the RA-degrading enzyme CYP26B1 can also lead to craniosynostosis (Laue et al. 2011). Recently, RA has been shown to increase gene expression of several hedgehog and bone morphogenetic protein (BMP) ligands, which might reveal the potential mechanisms of retinoic acid-induced craniosynostosis (James et al. 2010).

BMPs play key roles in skeletal developmental patterning, including osteoblast and chondrocyte differentiation, cartilage and bone formation, and craniofacial and limb development (Rahman et al. 2015; Matsui and Klingensmith 2014; Nie et al. 2006; Norrie et al. 2014). BMPs and their inhibitors are involved in the development of normal suture patency as well as the pathological suture fusion. BMP2 was highly expressed in the cells isolated from fused sutures of sagittal and coronal synostosis patients, while BMP4 was highly expressed in those of fused metopic or lambdoid synostosis (Whitton et al. 2016). The expression of BMP4 was elevated in the craniosynostotic sutural ligament and dura of coronal craniosynostotic rabbits (Rottgers et al. 2016). Study found that enhanced BMP signaling through a constitutively active form of the BMP type $1 \mathrm{~A}$ receptor (BMPR1A) in cranial neural crest cells caused premature suture fusion in mice (Komatsu et al. 2013). In a genome-wide association study for nonsyndromic sagittal craniosynostosis, susceptibility loci were found near BMP2 and within BBS9 (Bardet-Biedl syndrome 9), which were genes related with skeletal development (Justice et al. 2012).

In the present study, we test the hypothesis that BMP signaling is involved in retinoic acid-induced premature fusion in vitro and in vivo to better illustrate the molecular mechanisms of retinoic acid-induced premature suture fusion. Moreover, we explored the possibility of controlreleased Noggin from biocompatible PLGA microspheres in prevention of all-trans retinoic acid (atRA)-induced CS.

\section{Materials and methods}

\section{Specimen preparation}

Newborn C57BL/6J mice were purchased from the Medical Animal Center of Sun Yat-sen University (Guangzhou, China). For normal development of the suture, the skulls of mice at postnatal day 15 (P15), P20, P25, P30, P35, P40, and P45 was harvested. For subcutaneous injection model, mice were divided into two groups: control and atRA. atRA (R2625, Sigma-Aldrich, St. Louis, USA) was diluted in ethanol at $0.01 \mathrm{M}(3 \mathrm{mg} / \mathrm{ml})$, and approximately $10 \mu \mathrm{l}$ was delivered by subcutaneous injection into the area near the posterior frontal suture at P10 at a final dose of $100 \mathrm{mg} / \mathrm{kg}$ of mice weight. The skulls of the mice were harvested at P20. All skulls were fixed in 4\% paraformaldehyde (AR1068, Boster, Wuhan, China) at $4{ }^{\circ} \mathrm{C}$ prior to microCT scanning.

\section{Micro-computed tomography scanning and reconstruction}

Mouse skulls were scanned by high-resolution micro-computed tomography (microCT, Scanco Medical $\mu$ CT 50, Switzerland) with scanning parameters set to $70 \mathrm{kV}$ and $200 \mu \mathrm{A}$, a voxel size of $20 \mu \mathrm{m}$, and $1024 \times 1024$ resolution. The integration time per projection was $300 \mathrm{~ms}$. During scanning, the skulls were placed vertically into a polyethylene cylinder. Reconstruction and analysis of the skulls were performed using Avizo 8.1 (FEI Visualization Sciences Group).

\section{Histological staining}

After decalcification with 0.5 M EDTA for 1 month, the skulls were serially dehydrated with various concentrations of ethanol and xylene, embedded in paraffin, and sectioned $(5 \mu \mathrm{m}$ thick) according to standard procedures. Cross sections of the posterior frontal sutures were collected. Sections were deparaffinized and stained with hematoxylin and eosin 
(H\&E) to examine suture development of atRA-injected and control mice.

\section{Cell culture}

Isolation and culture of the suture-derived mesenchymal cell were performed as previously described (James et al. 2010; $\mathrm{Xu}$ et al. 2007). Five-day-old C57BL/6J pups $(n=$ 80) were purchased from the Medical Animal Center of Sun Yat-sen University (Guangzhou, China). The pups were sacrificed by decapitation, and posterior frontal sutures with 500- $\mu \mathrm{m}$ bony margins were dissected meticulously in sterile, cold phosphate-buffered saline (PBS, $\mathrm{pH}$ 7.2-7.4). To minimize tissue heterogeneity, sutures were separated from the underlying dura mater and overlying pericranium. Approximately 10 sutures were placed in a 6 -cm culture dish with the endocranial surface face down in $1 \mathrm{ml}$ of control medium (Dulbecco's modified Eagle's medium with $10 \%$ fetal bovine serum, $100 \mathrm{U} / \mathrm{ml}$ penicillin, and $100 \mathrm{U} / \mathrm{ml}$ streptomycin (Gibco, USA)) at $37{ }^{\circ} \mathrm{C}$ in a $5 \% \quad \mathrm{CO}_{2}$ atmosphere. Medium was replenished every 2 days. First-passage suture-derived mesenchymal cells were used for all cell culture experiments. After attachment, SMCs were supplied with either control medium (CM), CM with $1 \mu \mathrm{M}$ atRA (CR), osteogenic differentiation medium (OM, StemPro ${ }^{\circledR}$ Osteogenesis Differentiation Kit, Gibco, USA), or OM with $1 \mu \mathrm{M}$ atRA (OR). Medium was changed every other day. RNA and protein were collected at day 14 for expression analysis.

\section{Cell proliferation assay}

Logarithmic-phase SMCs were plated into 96-well plates at a density of 5000 cells/well in DMEM with $10 \%$ FBS, $100 \mathrm{U} / \mathrm{ml}$ penicillin, and $100 \mathrm{U} / \mathrm{ml}$ streptomycin. After $24 \mathrm{~h}$ of incubation, the medium was replaced with $\mathrm{CM}$, CR, OM, or OR. At days $0,1,2,3,4$, and 5, $10 \mu \mathrm{l}$ of CCK-8 (Dojindo, Japan) solution was added to each well. After an additional $1 \mathrm{~h}$ of incubation at $37^{\circ} \mathrm{C}$, absorbance was measured at $450 \mathrm{~nm}$ using a microplate reader (Infinite200, Tecan).

\section{Alkaline phosphatase assay and staining}

To determine ALP activity levels in SMCs, a colorimetric assay was performed using an ALP assay kit (Nanjingjiancheng, Jiangsu, China) according to the manufacturer's instructions. At days 7 and 14, cells were lysed in $0.1 \mathrm{~mol} / 1$ Triton X-100 (MP) for $1 \mathrm{~h}$ and centrifuged at $1000 \mathrm{rpm}$ for $10 \mathrm{~min}$, after which the supernatants were collected. P-Nitrophenylphosphate was used as a substrate to detect ALP activity. The optical density of the red product paranitrophenol was measured at $520 \mathrm{~nm}$ using a microplate reader (Infinite200, Tecan). The total protein concentration of cell lysates was measured using a BCA Protein Assay Kit (CWBio, Beijing, China). ALP activity was expressed as the release of $1 \mathrm{mg}$ p-nitrophenol per $15 \mathrm{~min}$ per microgram of total cellular protein. On days 7 and 14, ALP staining was performed using the Alkaline Phosphatase Staining Kit (Stemgent, San Diego, USA) following the manufacturer's guidelines. Alkaline phosphatase appeared red or purple under light microscopy.

\section{Alizarin red S staining}

Alizarin red staining was performed to detect extracellular mineralization. Briefly, cells were fixed with $4 \%$ paraformaldehyde at room temperature for $10 \mathrm{~min}$ and then incubated with $1 \%$ alizarin red S (MP, USA) for $1 \mathrm{~h}$, followed by extensive washing with distilled water. The staining of calcium mineral deposits of terminally differentiated cells was recorded using an inverted phase-contrast microscope (Axiovert 40, Zeiss).

\section{Quantitative real-time reverse transcription-polymerase chain reaction}

Total RNA was extracted using Trizol reagent (Invitrogen, USA). cDNA was synthesized using a Roche RT-PCR System (Roche). Real-time reverse transcription-polymerase chain reaction (RT-PCR) was performed using two-step RTPCR assays (Roche). Markers of osteogenic differentiation (runt-related transcription factor 2 (Runx2), alkaline phosphatase (ALP), type I collagen (Colla1), osteopontin (OPN), and osteocalcin (OC)) and markers of BMP signaling (BMPR-1a, BMPR-1b, BMPR-2, BMP2, BMP4, Noggin, smad1, smad5, and smad9 (also known as Smad8)) were examined. The specific primers used for detecting mRNA transcripts are shown in Table 1. Transcripts were normalized according to the $\beta$ actin transcript levels and compared with the control using the $2^{- \text {ddCt }}$ method.

\section{Preparation of Noggin-encapsulated microspheres with PLGA and in vivo treatment}

Microspheres of poly (d-1-lactic-co-glycolic acid) (PLGA, Sigma, St. Louis, MO) of 50:50 were prepared as described before. Briefly, $250 \mathrm{mg}$ PLGA was dissolved into $1 \mathrm{ml}$ chloromethane, and $50 \mu \mathrm{l}$ of recombinant Noggin (R\&D Systems, Minneapolis, MN) or the control bovine serum albumin was mixed with $1 \mathrm{ml}$ PLGA solution. Then, $2 \mathrm{ml}$ of $1 \%$ polyvinyl alcohol (PVA) was added in PLGA solution, followed by 1 -min mixing. A total of $100 \mathrm{ml}$ of $2 \%$ isopropanol was added to the final emulsion and stirred for $2 \mathrm{~h}$. Microspheres were stored in liquid nitrogen and lyophilized for at least $48 \mathrm{~h}$ before use. Control release kinetics of 
Table 1 Primer pairs used for RT-PCR amplifications

\begin{tabular}{|c|c|}
\hline Gene & Primer \\
\hline BMPR-1A & $\begin{array}{l}\text { F: GGCCATTGCTTTGCCATTAT } \\
\text { R: CGGTGAATCCTTGCATTGAAA }\end{array}$ \\
\hline BMPR-1B & $\begin{array}{l}\text { F: GGACATGCTGGACTTGGCTTC } \\
\text { R: TTATTAGGGACTTGTGAGCCTGGAC }\end{array}$ \\
\hline BMPR-2 & $\begin{array}{l}\text { F: GAGCCAGACGGCAAGAGCTTA } \\
\text { R: TCGCTTCATAGTTGGAGACGAGAG }\end{array}$ \\
\hline Smad1 & $\begin{array}{l}\text { F: GGAATGCTGTGAGTTCCCATTTG } \\
\text { R: TGCTGAGGATTGTACTCGCTGTG }\end{array}$ \\
\hline Smad5 & $\begin{array}{l}\text { F: AGACCATGCCCAGCATATCCA } \\
\text { R: TTGACAACAATCCCAGGCAGAA }\end{array}$ \\
\hline Smad9 & $\begin{array}{l}\text { F: AGGTCTGCATCAACCCATACCATTA } \\
\text { R: ACTTCGGAACTTGGCCAGGAG }\end{array}$ \\
\hline Noggin & $\begin{array}{l}\text { F: GAAGTTACAGATGTGGCTGTGGTC } \\
\text { R: AAGCAGCTGCCCACCTTCA }\end{array}$ \\
\hline BMP2 & $\begin{array}{l}\text { F: TGACTGGATCGTGGCACCTC } \\
\text { R: CAGAGTCTGCACTATGGCATGGTTA }\end{array}$ \\
\hline BMP4 & $\begin{array}{l}\text { F: TTTGTTCAAGATTGGCTCCCAAG } \\
\text { R: AAACGACCATCAGCATTCGGTTA }\end{array}$ \\
\hline Runx2 & $\begin{array}{l}\text { F: CCAGAATGATGGTGTTGACG } \\
\text { R: GGTTGCAAGATCATGACTAGG }\end{array}$ \\
\hline ALP & $\begin{array}{l}\text { F: GGGACGAATCTCAGGGTACA } \\
\text { R: AGTAACTGGGGTCTCTCTCTTT }\end{array}$ \\
\hline OPN & $\begin{array}{l}\text { F: TACGACCATGAGATTGGCAGTGA } \\
\text { R: TATAGGATCTGGGTGCAGGCTGTAA }\end{array}$ \\
\hline $\mathrm{OC}$ & $\begin{array}{l}\text { F: GGGCAATAAGGTAGTGAACAGACT } \\
\text { R: CAAGCAGGGTTAAGCTCACACT }\end{array}$ \\
\hline Colla1 & $\begin{array}{l}\text { F: GACATGTTCAGCTTTGTGGACCTC } \\
\text { R: GGGACCCTTAGGCCATTGTGTA }\end{array}$ \\
\hline$\beta$-Actin & $\begin{array}{l}\text { F: CATCCGTAAAGACCTCTATGCCAAC } \\
\text { R: ATGGAGCCACCGATCCACA }\end{array}$ \\
\hline
\end{tabular}

Noggin from the PLGA microsphere in vitro was analyzed by the ELISA kit as instructed.

\section{Treatment of recombinant mouse Noggin in vitro and in vivo}

SMCs were cultured in OR with or without recombinant mouse Noggin (200 ng/ml, RD) for 21 days for alizarin red staining. For in vivo analysis of the efficacy of the microsphere based therapy, atRA-induced CS mice were injected with $2 \mathrm{mg}$ Noggin- or BSA-encapsulated PLGA microspheres dissolved in $20 \mu \mathrm{L}$ collagen gel 1 day post atRA injection. Mouse skulls were harvested at P20 for microCT scanning.

\section{Statistical analysis}

All experiments were performed at least three times. All quantitative data are expressed as the mean \pm standard deviation and were analyzed using Student's $t$ test or one-way ANOVA followed by a post hoc Tukey range test in GraphPad Prism, with significance set at $P<0.05$. In figures, bars in graphs represent means, and error bars represent $1 \mathrm{SD}$.

\section{Results}

atRA caused premature fusion of the posterior frontal suture in a subcutaneous injection mouse model

As the posterior frontal suture is the only calvarial suture fuses physiologically, precise course of the fusing time was analyzed from P15-P45. The posterior frontal suture fused from 20 and 45 days postnatal at the ectocranial layer from anterior to posterior, while the endocranial layer remains open till P45 (Fig. 1a). To establish a mouse model of atRA-induced craniosynostosis, atRA was injected into the area near the posterior frontal suture subcutaneously. In the subcutaneous injection assay, the posterior frontal sutures of P20 mice in the atRA-injected group $(n=6)$ fused prematurely, while those in control mice $(n=6)$ developed normally. The trabeculae in these fusion sutures were irregularly arranged in both cross sections of 3D reconstruction and H\&E staining (Fig. 1b). We quantified the skull morphology of both control and atRAinjected mice using anatomical landmarks representing the entire skull and the facial skeleton. No significant differences were found in skull length, skull width, skull height, nasal length, maxilla length, mandible length, or interorbital distance between the atRA-injected mice $(n=6)$ and the control group $(n=6)$ (Fig. 1c).

\section{atRA repressed the proliferation of SMCs}

To determine the effect of either atRA or osteogenic medium on SMC proliferation, cell proliferation assay was performed at days $0,1,2,3,4$, and 5 (Fig. 2). The OD value increased significantly in osteogenic differentiation medium (OM and $\mathrm{OR})$ compared with that in control medium (CM) at day 1, after which the OD plateaued. After atRA treatment, the OD values of cells in the $\mathrm{CR}$ and $\mathrm{OR}$ groups decreased significantly after day 1 in comparison with those in the corresponding control groups $(P<0.05)$.

Fig. 1 atRA caused premature fusion of posterior frontal suture in a subcutaneous injection mouse model. a Time course of the development of the suture in normal mice. b 3D reconstruction and H\&E staining of the morphology of posterior frontal suture in atRAinjected and control mice. The posterior frontal sutures of P20 mice in the atRA-injected group $(n=6)$ fused prematurely, while those in control mice $(n=6)$ continued to develop normally. $\mathbf{c}$ Measurements of the skull and facial skeleton of atRA-injected and control mice. No significant differences were found in skull length, skull width, skull height, nasal length, maxilla length, mandible length, or interorbital distance between the atRA-injected mice $(n=6)$ and the control group $(n=6)$ 
a
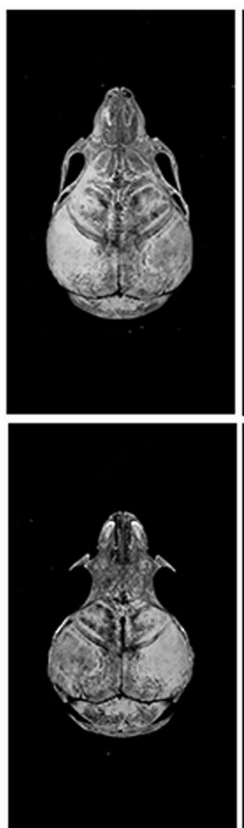

P15
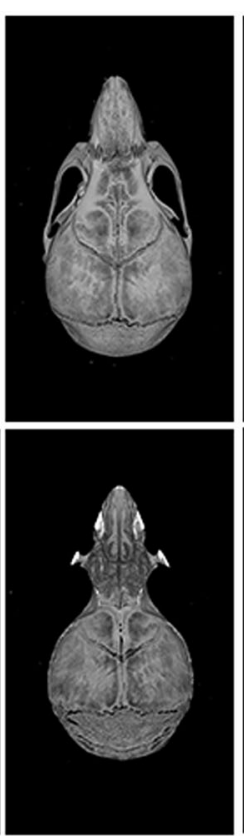

P20
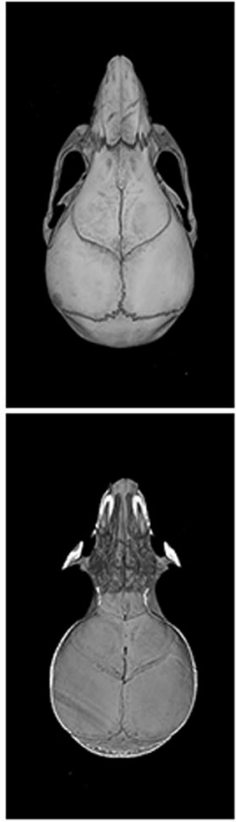

P25
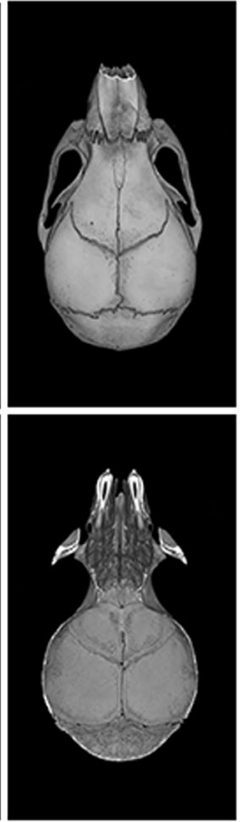

P30
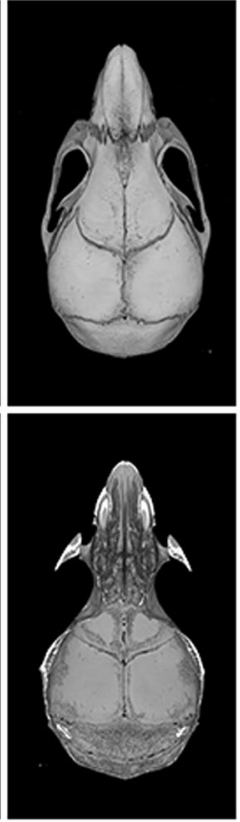

P35
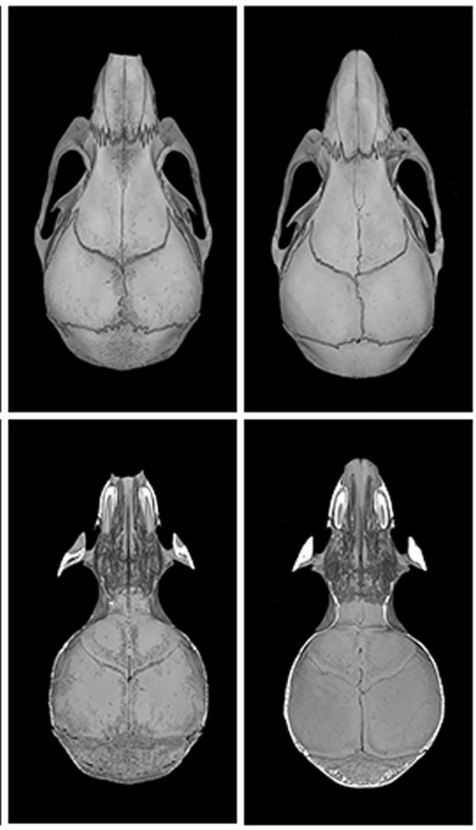

P40

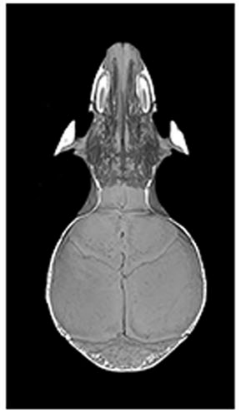

P45
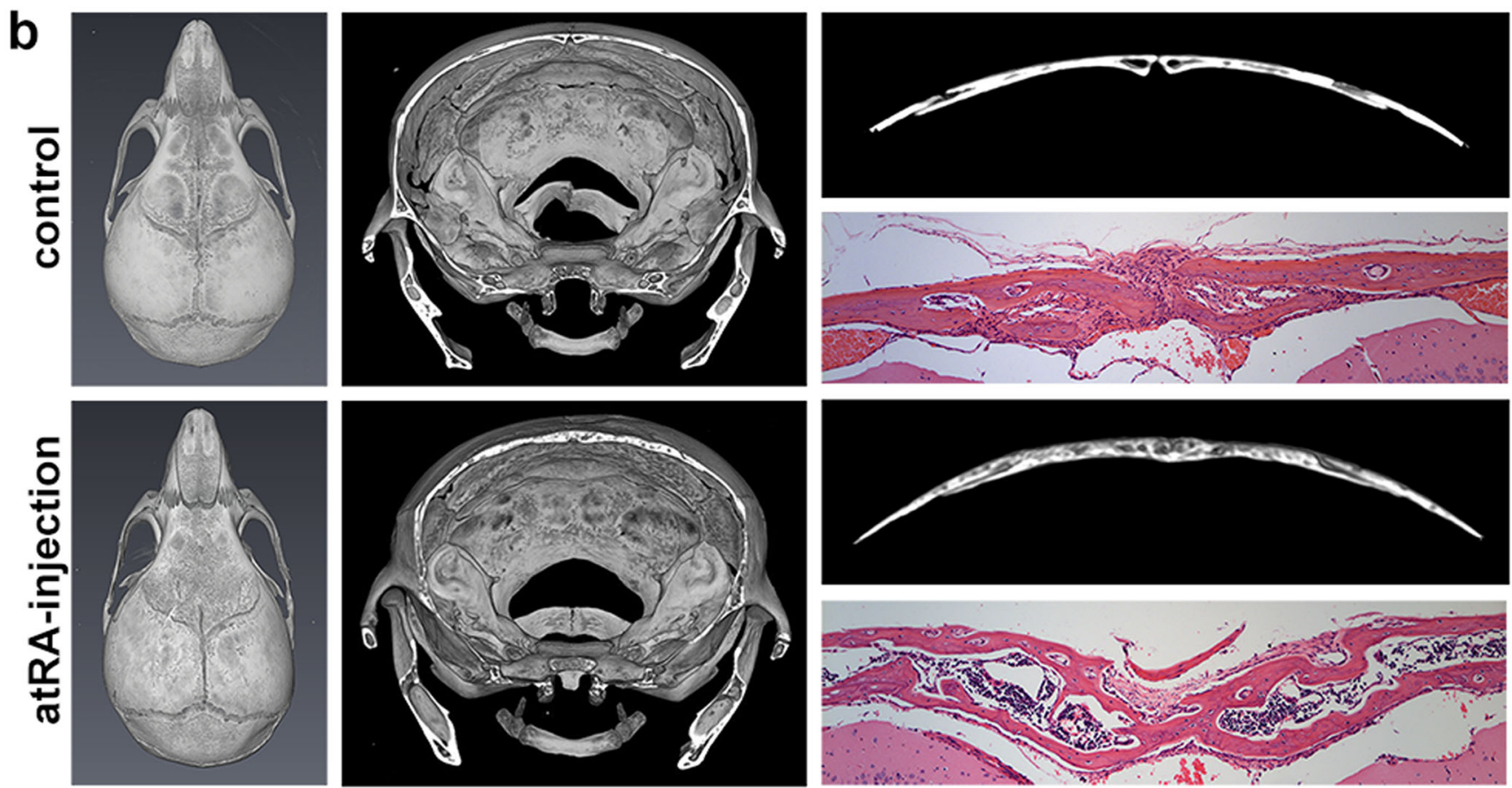

C
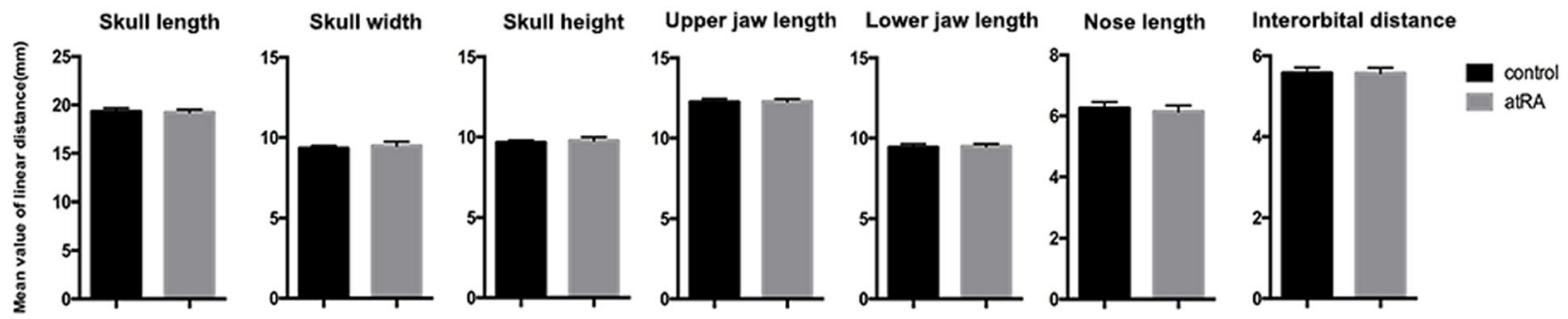


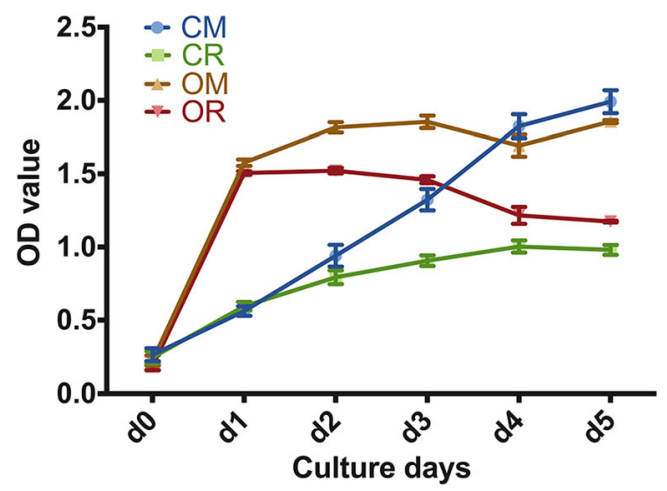

Fig. 2 Cell proliferation of SMCs in response to different media. Osteogenic medium increased the proliferation of cells in OM and OR at day 1. atRA decreased the proliferation of cells in the CR and OR groups after day 1 when compared with the corresponding control groups $(P<0.05)$. Values represent calculated means and are normalized to culture in $\mathrm{CM}$. Error bars $=1 \mathrm{SD} ; n=3 ; * P<0.05$

\section{atRA enhanced in the osteogenic capacity of SMCs}

To identify the effect of either atRA or osteogenic medium on ALP activity in SMCs, ALP staining and ALP activity assay was detected at days 7 and 14. Strong ALP staining was observed in the OR groups at both day 7 and day 14 , whereas the $\mathrm{OM}$ and $\mathrm{CR}$ groups demonstrated weaker alkaline phosphatase staining, and no staining was observed in the CM groups (Fig. 3a). The results of ALP activity were consistent with those of the ALP staining at day 7 and day 14. Osteogenic differentiation medium induced mesenchymal cells to exhibit increased ALP activity in OM and OR cultures at days 7 and 14 compared with the corresponding control groups in CM, whereas CR did not significantly increase ALP activity. The maximal expression level of ALP activity was observed in cells cultured in OR $(P<0.05)$, which was approximately 20- and 8-fold greater than the levels measured in the CM cultures at days 7 and 14, respectively. Comparing the ALP activity between the OM and OR groups, the OR group showed significantly higher ALP activity than the OM group at day $14(P<0.05)$, whereas ALP activity in OR and OM did not differ at day $7(P>0.05)$ (Fig. $3 \mathrm{~b})$. The above results suggested that osteogenic medium increased ALP activity in SMCs and that these effects were enhanced with atRA supplementation of osteogenic medium.

To detected extracellular mineralization, mineralized nodules of SMCs in the four different media were evaluated at 21 days of culture using alizarin red S staining. In OM, mineralized nodules were present at 21 days in culture, and this mineralization increased with the supplementation of atRA, whereas there were no bone nodules in CM or CR (Fig. 3c). Accordingly, several osteoblast markers, the mRNA level of Runx2, Alp, Col1a1, Opn, and Oc, were significantly upregulated at 14 days in osteogenic differentiation medium with atRA supplementation $(P<0.05)$ (Fig. 3d). In particular, Alp
mRNA levels in OR were increased 60-fold compared with $\mathrm{CM}$ and 12-fold compared with OM.

\section{atRA enhanced the osteogenic capacity of SMCs via BMP-SMAD pathway}

Since BMP signaling has been shown to promote osteogenic differentiation of several cell lines, we evaluated the mRNA levels of genes related to BMP signaling. The mRNA expression of Bmpr-2 was observed to be significantly $(P<0.05)$ upregulated, by approximately 3.5 -fold, in cells cultured in OR compared with $\mathrm{CM}$ or OM at day 14 , while the transcript levels of Bmpr-1a and Bmpr-1b were also increased but not significantly $(P>0.05)$ (Fig. 4a-c). The relative mRNA level of Bmp4 increased approximately 2 -fold in cells cultured in OR compared with that in $\mathrm{CM}$ or OM, while that of Bmp2 remained unchanged (Fig. 4d, e). Smad1, Smad5, and Smad9 were measured because they are immediate downstream effectors in the BMP signaling pathway. Smad9 mRNA expression was upregulated at day 14 under OM and OR culture conditions $(P<0.05)$. However, Smad1 and Smad5 mRNA levels were only modestly upregulated in the cells cultured in OR compared to those cultured in $\mathrm{CM}(P>0.05)$ (Fig. $4 \mathrm{f}-\mathrm{h})$. These results suggested that atRA might enhance the osteogenic differentiation of SMCs at least in part through upregulation of BMP/Smad signaling.

\section{PLGA microsphere-based Noggin control release blocks the atRA-induced suture fusion}

Noggin inactivates the BMP signaling pathway by competing with receptors for ligand binding. To test the effects of Noggin in vitro, SMCs were cultured in OR with or without recombinant mouse Noggin. After 21 days of induction, alizarin red staining showed that recombinant mouse Noggin inhibited the atRA-induced enhancement of osteogenesis of SMCs, with many fewer mineralized nodules present in the Noggintreated group (Fig. 5a). We then tested whether control release of Noggin would block the atRA-induced suture fusion in vivo. First, Noggin or control BSA was encapsulated into the PLGA microspheres (Fig. 5b), which was confirmed by a scanning electron microscope (Fig. 5c). Release kinetics further confirmed the control release of Noggin from the PLGA microspheres (Fig. 5d). Next, 1day post atRA-induced CS, mice were tropically injected with $2 \mathrm{mg}$ Noggin- or BSA-encapsulated microspheres dissolved in $20 \mu \mathrm{L}$ collagen gel. As shown in Fig. 5e, the fusion of posterior frontal suture was partially blocked in Noggin-injected mice, while those injected with Noggin-encapsulated PLGA microspheres were much more obvious than those of naked Noggin. 
a

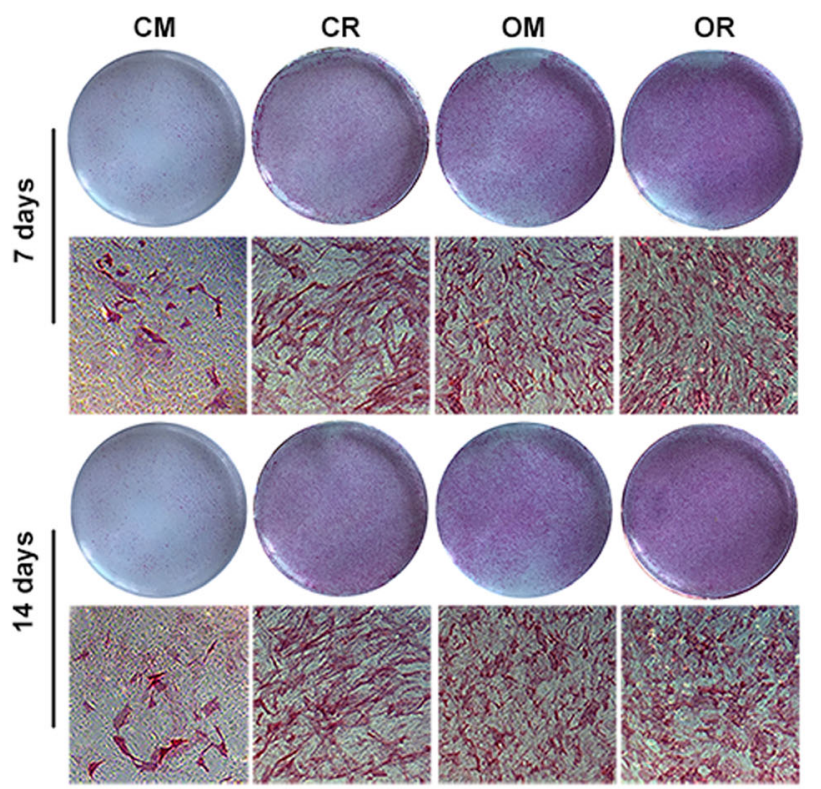

b

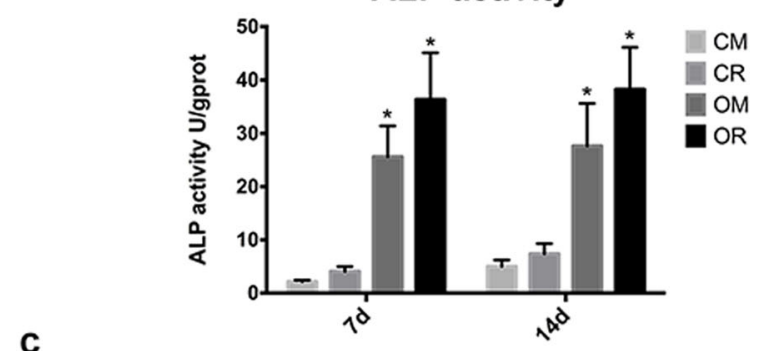

d
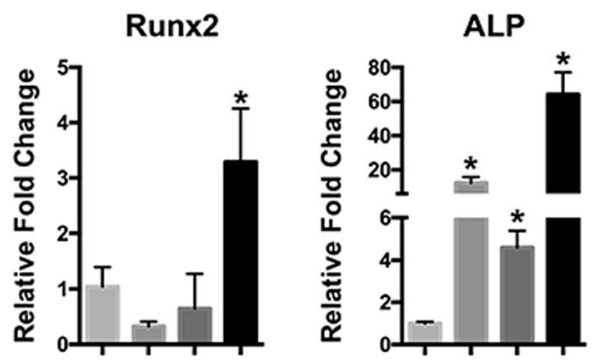

Fig. 3 atRA increased osteogenesis in SMCs. atRA increased alkaline phosphatase staining (a) and alkaline phosphatase activity (b) at day 7 and day 14 of culture in differentiation media. $\mathbf{c}$ atRA enhanced mineralization in SMCs as measured by alizarin red S staining. $\mathbf{d}$ atRA increased the mRNA level of markers related to osteogenesis. Runx2,
Col1a1

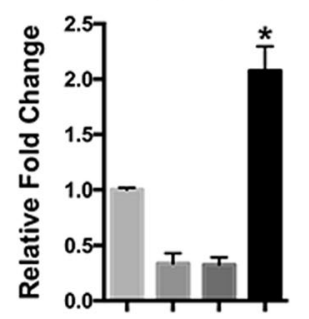

OPN

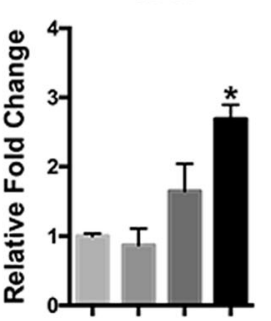

OC

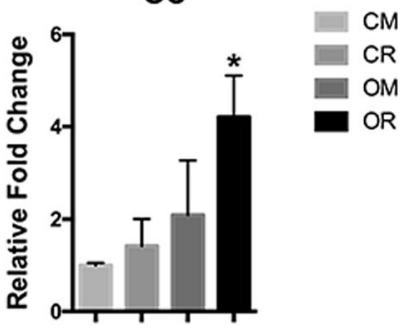

ALP, Colla1, OPN, and OC were significantly upregulated at day 14 in osteogenic differentiation medium supplemented with atRA $(P<0.05)$. Values represent calculated means and are normalized to culture in CM. Error bars $=1 \mathrm{SD} ; n=3 ; * P<0.05$

\section{Discussion}

Retinoic acid, a well-known teratogen, has been shown to be involved in craniosynostosis (CS). However, the mechanisms by which retinoic acid causes craniosynostosis are unclear yet. In the current study, we for the first time revealed that excessive BMP signal pathway is essential for atRA-induced CS. Moreover, we also revealed that PLGA microsphere encapsulated with Noggin, an inhibitor of BMP pathway, significantly prevented and partially restored the atRA-induced suture fusion in vivo.

BMPs are key growth factors in skeletal differentiation that interact with bone morphogenetic protein receptors (BMPRs) on the cell surface to phosphorylate the transcription factors Smad1/5/9, thereby allowing them to bind with Smad4; the Smad complex then activates BMP target genes (Rahman et al. 2015; Wu et al. 2016; Miyazono et al. 2010; Sieber et al. 2009). BMP signaling

pathways play important roles during embryonic development and early skeletal formation, and abnormal BMP signaling can cause skeletal disorders (Bandyopadhyay et al. 2013; Li and Cao 2006; Rosen 2006; Yamamoto and Oelgeschlager 2004). Recombinant human BMP4 induced significant changes in alkaline phosphatase activity in a dose-dependent manner in cells derived from either wild-type or craniosynostotic rabbits (Cooper et al. 2010). Transgenic mice expressing a truncated dominantnegative BMPR-1B targeted to osteoblasts using the type I collagen promoter showed impairment of postnatal bone formation, and their bone mineral density, bone volume, and bone formation rate were severely reduced (Zhao et al. 2002). Mice with a conditional knockdown of BMPR-1A had wide-open anterior fontanelles and developed short faces, hypertelorism, and calvarial foramina (Saito et al. 2012). Another study showed that enhanced BMP signaling through the BMPR-1A in cranial neural 
a

BMPR1A

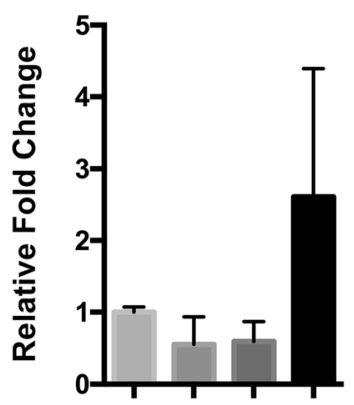

e

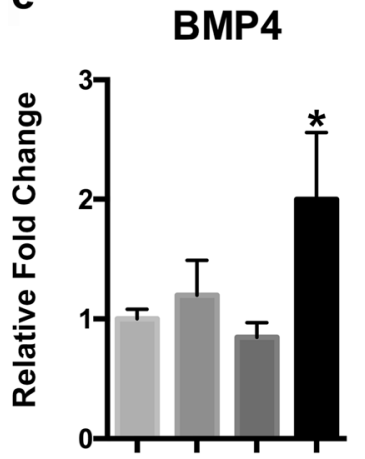

b

BMPR1B

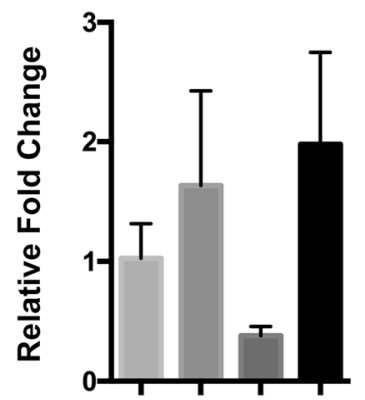

$\mathbf{f}$

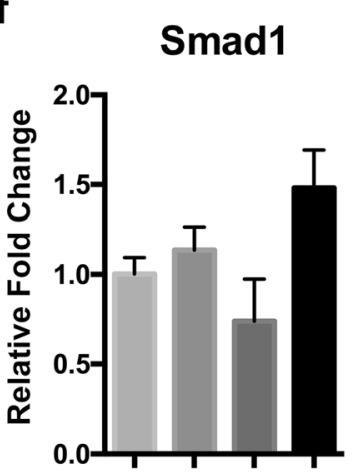

C

BMPR2

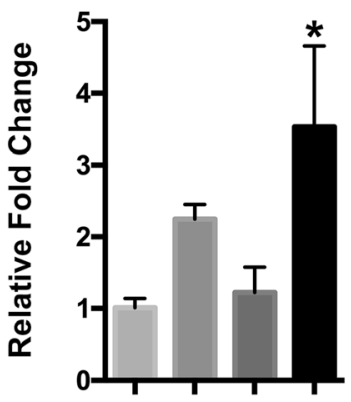

g

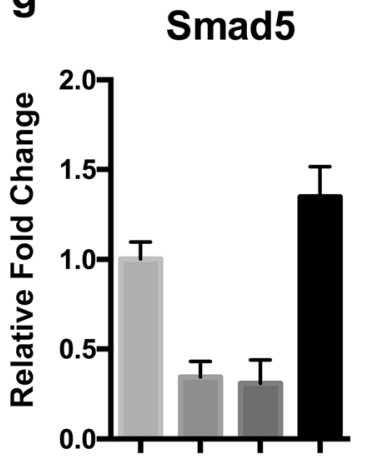

d

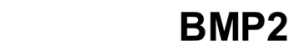

Fig. 4 atRA increases the mRNA and protein expression of BMP signaling components in SMCs. The mRNA expression levels of Bmpr1A (a), Bmpr-1B (b), Bmpr-2 (c), Bmp2 (d), Bmp4 (e), Smad1 (f),

Smad5 (g), and Smad9 (h) in samples as indicated were analyzed by qPCR $(P<0.05)$. Error bars $=1 \mathrm{SD} ; n=3 ; * P<0.05$

crest cells caused premature suture fusion in mice (Komatsu et al. 2013). As for BMPR-2, there were controversies of the effect of BMPR-2 on the development of the skeletogenesis as well. Gamer (Gamer et al. 2011) found that loss of BMPR-2 in the early limb has no effect on limb skeletal patterning or endochondral ossification, whereas BMPR-2 mutants displayed delayed ossification in the lateral ossification centers of vertebrae and the interparietal bone (Delot et al. 2003; Yang et al. 2010). Recently, Smad9 was demonstrated to be a new type of transcriptional regulator in BMP signaling, and its expression increased when cells exposed to BMP4 (Tsukamoto et al. 2014). Our study here further revealed that the BMP-SMAD pathway is involved in atRA-induced suture fusion. Our study showed that BMP pathway genes, in particular mRNAs for BMPR-2, BMP4, and Smad9, were abundantly expressed in SMCs, which were further enhanced by atRA, suggesting that atRA might increase the osteogenic differentiation capacity of SMCs by transcriptionally regulating the BMP-SMAD pathway.

In light of the involvement of BMP-SMAD pathway, we further explored whether inhibition of BMP pathway would prevent the premature fusion. Noggin was selected based on the findings that Noggin could block BMP function by binding BMP-2, BMP-4, BMP-5, BMP-6, and

BMP-7 with various degrees of affinity (Canalis et al. 2003). As expected, we revealed that control release of Noggin via PLGA microspheres significantly blocked the fusion in atRA-induced craniosynostosis in mice. The intervention experiments further concluded that atRA induces CS via BMP pathway. Moreover, the study also provided a delivery strategy and a drug candidate for CS prevention. The finding here is consistent with the previous studies on the role of Noggin. For example, Warren has found that ectopic noggin expression prevented the fusion of mouse posterior frontal sutures (Warren et al. 2003). In rabbit and rat models of coronal suture synostosis, Noggin was found to inhibit bone healing and delay resynostosis at the surgical site after suturectomy (Cooper et al. 2007; Shen et al. 2009). In a later study, Noggin was proved to be no effect on the initial suture fusion of a delayed-onset coronal suture synostosis rabbit model (Cray et al. 2011). Currently, treatments for craniosynostosis include suturectomy, minimally invasive craniectomy, and spring-mediated distraction osteogenesis (Gasparini et al. 2012; Okada and Gosain 2012); however, there are high rates of mortality and resynostosis in surgery, and distraction osteogenesis has limited effects on the cranial vault (Hermann et al. 2013). The proposed control release of Noggin via PLGA 


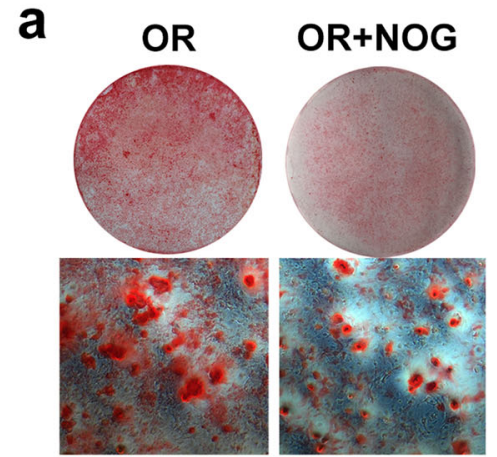

C
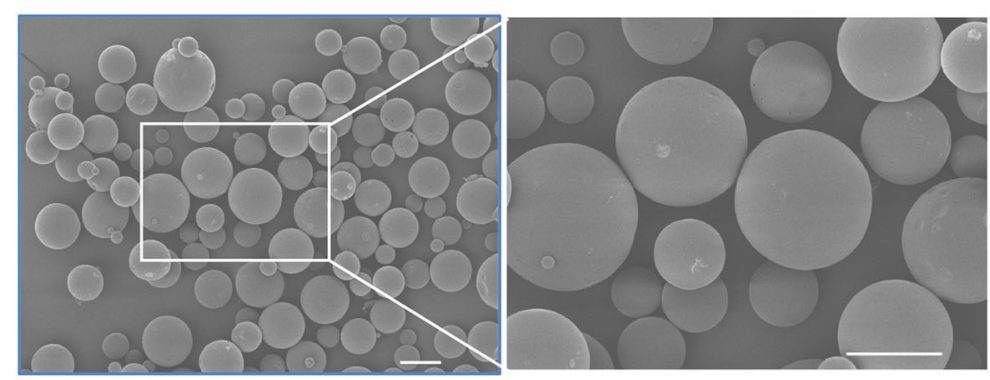

b

PLGA

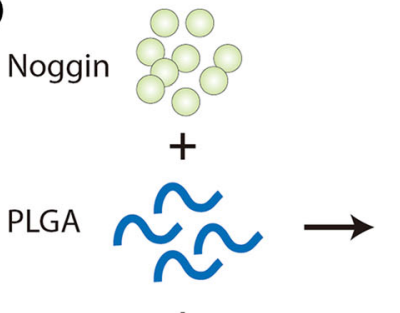

PVA

$+$

PLGA microsphere

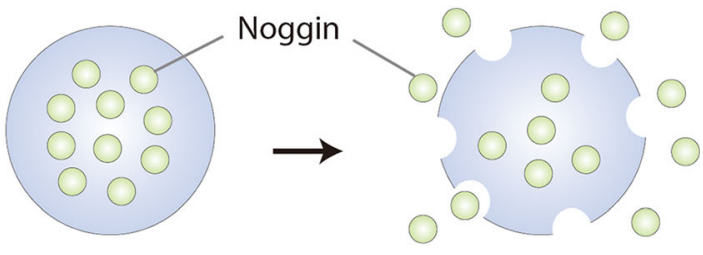

Control release of Noggin

$\mathbf{e}$

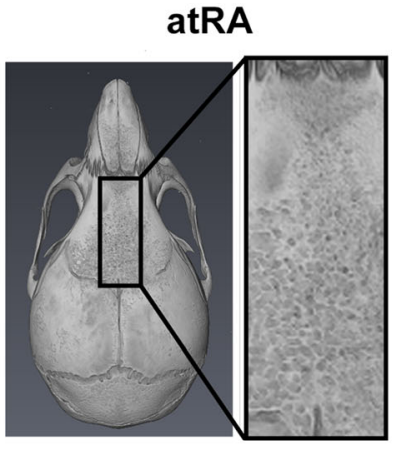

Fig. 5 Noggin delivery blocked the atRA-induced suture fusion. a Recombinant mouse Noggin inhibited the atRA-induced enhancement of osteogenesis of SMCs as measured using alizarin red staining. b Schematic representation of Noggin encapsulation in PLGA microspheres. c The scanning electron microscope image of PLGA
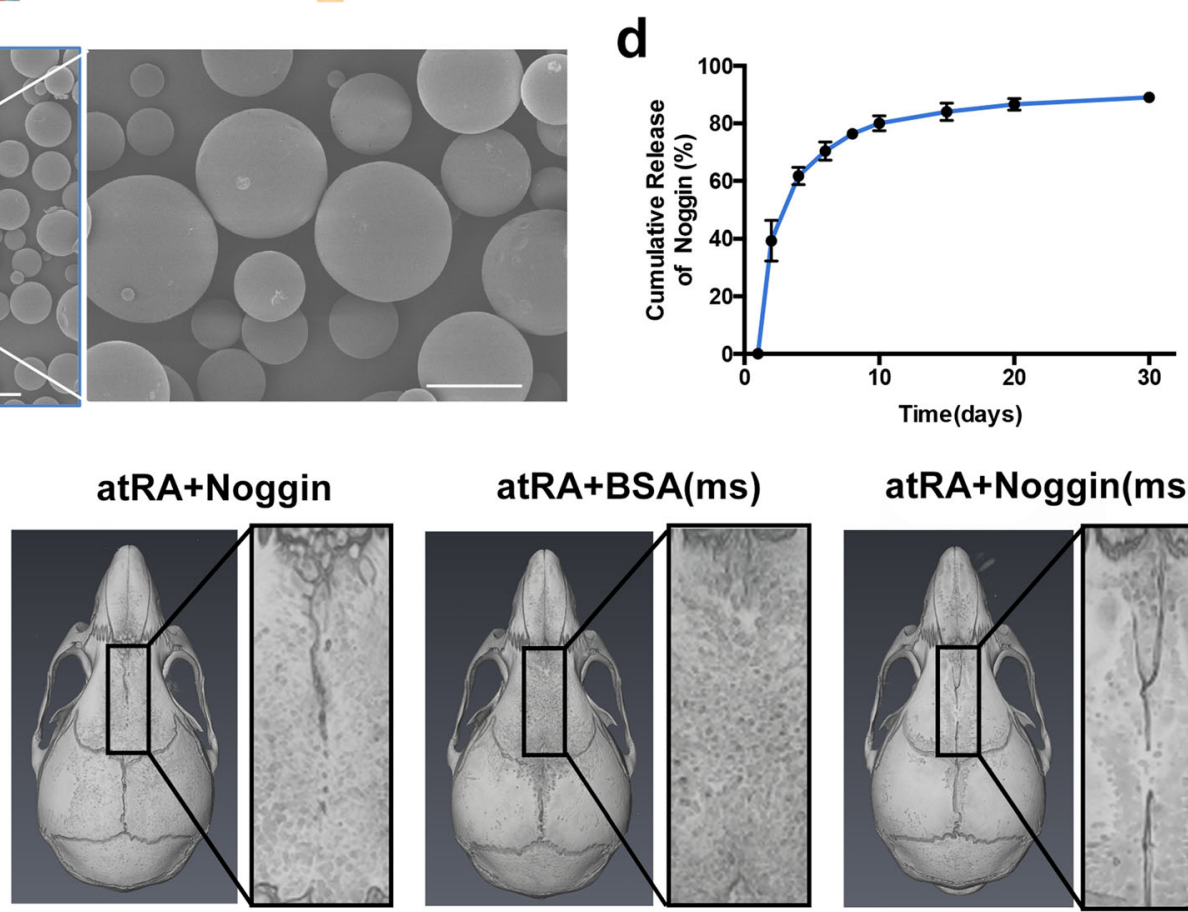

microspheres. d Release kinetics further confirmed the control release of Noggin from the PLGA microspheres. e Representative image of the fusion of the posterior frontal suture in indicated groups $(n=6$ for each group)

fuses at 25-45 days of age, while the other calvarial sutures remain patent through life (Bradley et al. 1996). Recent study found that the endocranial layer of PF suture fusion in CD1 wild-type mice occurs from approximately postnatal days 7 to 15 , while the ectocranial layer remains patent until P25 (Sahar et al. 2005).

In summary, we here revealed that excessive BMP signal pathway is essential for atRA-induced CS, while PLGA microsphere encapsulated with Noggin significantly prevented and partially restored the atRA-induced suture fusion in vivo. These data suggest that PLGA microsphere-based control release of Noggin emerges as a promising strategy for prevention of CS related to atRAinduced suture fusion. 
Funding This work was partially supported by Grants from the Project of the National Natural Science Foundation of China (Grant No. 81300862, No. 81600824); the Project of Natural Science Foundation of Guangdong Province, China (Grant No. 2015A030313880); the Project of Young Teachers training program of Sun Yat-sen University (Grant No. 16ykpy46, No. 17ykpy73); the Project of Science and Technology Program of Guangzhou (Grant No. 201707010106 , No. 201804010459); and the Project of National Key R\&D Program of China (Grant No. 2016YFC20160905200).

\section{Compliance with ethical standards}

Conflict of interest The authors declare that they have no conflicts of interest.

Ethical approval All animal procedures were performed in accordance with the Guidelines for the Care and Use of Laboratory Animals of Sun Yat-sen University and were approved by the Institutional Animal Care and Use Committee (IACUC) of Sun Yat-sen University (approval number: IACUC-DB-15-0104).

Open Access This article is distributed under the terms of the Creative Commons Attribution 4.0 International License (http:// creativecommons.org/licenses/by/4.0/), which permits unrestricted use, distribution, and reproduction in any medium, provided you give appropriate credit to the original author(s) and the source, provide a link to the Creative Commons license, and indicate if changes were made.

\section{References}

Bandyopadhyay A, Yadav PS, Prashar P (2013) BMP signaling in development and diseases: a pharmacological perspective. Biochem Pharmacol 85(7):857-864. https://doi.org/10.1016/j.bcp.2013.01. 004

Bradley JP, Levine JP, Roth DA, McCarthy JG, Longaker MT (1996) Studies in cranial suture biology: IV. Temporal sequence of posterior frontal cranial suture fusion in the mouse. Plast Reconstr Surg 98(6): 1039-1045

Canalis E, Economides AN, Gazzerro E (2003) Bone morphogenetic proteins, their antagonists, and the skeleton. Endocr Rev 24(2): 218-235. https://doi.org/10.1210/er.2002-0023

Carmichael SL, Ma C, Rasmussen SA, Honein MA, Lammer EJ, Shaw GM, National Birth Defects Prevention S (2008) Craniosynostosis and maternal smoking. Birth Defects Res A Clin Mol Teratol 82(2): 78-85. https://doi.org/10.1002/bdra.20426

Cooper GM, Curry C, Barbano TE, Burrows AM, Vecchione L, Caccamese JF, Norbutt CS, Costello BJ, Losee JE, Moursi AM, Huard J, Mooney MP (2007) Noggin inhibits postoperative resynostosis in craniosynostotic rabbits. J Bone Miner Res 22(7): 1046-1054. https://doi.org/10.1359/jbmr.070410

Cooper GM, Lensie EL, Cray JJ Jr, Decesare GE, Smalley MA, Losee JE, Mooney MP (2010) BMP-4 response in wild-type and craniosynostotic rabbit bone cells. Plast Reconstr Surg 125(5): 1403-1411. https://doi.org/10.1097/PRS.0b013e3181d62ad4

Coussens AK, Wilkinson CR, Hughes IP, Morris CP, van Daal A, Anderson PJ, Powell BC (2007) Unravelling the molecular control of calvarial suture fusion in children with craniosynostosis. BMC Genomics 8:458. https://doi.org/10.1186/1471-2164-8-458

Cray J Jr, Burrows AM, Vecchione L, Caccamese JF Jr, Losee JE, Moursi AM, Siegel MI, Cooper GM, Mooney MP (2011) Blocking bone morphogenetic protein function using in vivo noggin therapy does not rescue premature suture fusion in rabbits with delayed-onset craniosynostosis. Plast Reconstr Surg 127(3):1163-1172. https:// doi.org/10.1097/PRS.0b013e318205f23b

Delot EC, Bahamonde ME, Zhao M, Lyons KM (2003) BMP signaling is required for septation of the outflow tract of the mammalian heart. Development 130(1):209-220

Gamer LW, Tsuji K, Cox K, Capelo LP, Lowery J, Beppu H, Rosen V (2011) BMPR-II is dispensable for formation of the limb skeleton. Genesis 49(9):719-724. https://doi.org/10.1002/dvg.20761

Gardner JS, Guyard-Boileau B, Alderman BW, Fernbach SK, Greene C, Mangione EJ (1998) Maternal exposure to prescription and nonprescription pharmaceuticals or drugs of abuse and risk of craniosynostosis. Int J Epidemiol 27(1):64-67

Gasparini G, Di Rocco C, Tamburrini G, Pelo S (2012) External craniofacial osteodistraction in complex craniosynostoses. Childs Nerv Syst 28(9):1565-1570. https://doi.org/10.1007/s00381-012-1820-y

Hermann CD, Lawrence K, Olivares-Navarrete R, Williams JK, Guldberg RE, Boyan BD, Schwartz Z (2013) Rapid re-synostosis following suturectomy in pediatric mice is age and location dependent. Bone 53(1):284-293. https://doi.org/10.1016/j.bone.2012.11. 019

Honein MA, Rasmussen SA (2000) Further evidence for an association between maternal smoking and craniosynostosis. Teratology 62(3): 145-146. https://doi.org/10.1002/1096-9926(200009)62:3<145:: AID-TERA1>3.0.CO;2-7

James AW, Levi B, Xu Y, Carre AL, Longaker MT (2010) Retinoic acid enhances osteogenesis in cranial suture-derived mesenchymal cells: potential mechanisms of retinoid-induced craniosynostosis. Plast Reconstr Surg 125(5):1352

Jentink J, Loane MA, Dolk H, Barisic I, Garne E, Morris JK, de Jong-van den Berg LT, Group EASW (2010) Valproic acid monotherapy in pregnancy and major congenital malformations. N Engl J Med 362(23):2185-2193. https://doi.org/10.1056/NEJMoa0907328

Johnson D, Wilkie AO (2011) Craniosynostosis. Eur J Hum Genet 19(4): 369-376. https://doi.org/10.1038/ejhg.2010.235

Justice CM, Yagnik G, Kim Y, Peter I, Jabs EW, Erazo M, Ye X, Ainehsazan E, Shi L, Cunningham ML, Kimonis V, Roscioli T, Wall SA, Wilkie AO, Stoler J, Richtsmeier JT, Heuze Y, SanchezLara PA, Buckley MF, Druschel CM, Mills JL, Caggana M, Romitti PA, Kay DM, Senders C, Taub PJ, Klein OD, Boggan J, Zwienenberg-Lee M, Naydenov C, Kim J, Wilson AF, Boyadjiev SA (2012) A genome-wide association study identifies susceptibility loci for nonsyndromic sagittal craniosynostosis near BMP2 and within BBS9. Nat Genet 44(12):1360-1364. https://doi.org/10. 1038/ng.2463

Kallen K (1999) Maternal smoking and craniosynostosis. Teratology 60(3):146-150. https://doi.org/10.1002/(SICI)1096-9926(199909) 60:3<146::AID-TERA10>3.0.CO;2-3

Komatsu Y, Yu PB, Kamiya N, Pan H, Fukuda T, Scott GJ, Ray MK, Yamamura K, Mishina Y (2013) Augmentation of Smad-dependent BMP signaling in neural crest cells causes craniosynostosis in mice. J Bone Miner Res 28(6):1422-1433. https://doi.org/10.1002/jbmr. 1857

Laue K, Pogoda HM, Daniel PB, van Haeringen A, Alanay Y, von Ameln S, Rachwalski M, Morgan T, Gray MJ, Breuning MH, Sawyer GM, Sutherland-Smith AJ, Nikkels PG, Kubisch C, Bloch W, Wollnik B, Hammerschmidt M, Robertson SP (2011) Craniosynostosis and multiple skeletal anomalies in humans and zebrafish result from a defect in the localized degradation of retinoic acid. Am J Hum Genet 89(5):595-606. https://doi.org/10.1016/j.ajhg.2011.09.015

Leitch VD, Dwivedi PP, Anderson PJ, Powell BC (2013) Retinol-binding protein 4 downregulation during osteogenesis and its localization to non-endocytic vesicles in human cranial suture mesenchymal cells suggest a novel tissue function. Histochem Cell Biol 139(1):75-87. https://doi.org/10.1007/s00418-012-1011-7

Li X, Cao X (2006) BMP signaling and skeletogenesis. Ann N Y Acad Sci 1068:26-40. https://doi.org/10.1196/annals.1346.006 
Matsui M, Klingensmith J (2014) Multiple tissue-specific requirements for the BMP antagonist Noggin in development of the mammalian craniofacial skeleton. Dev Biol 392(2):168-181. https://doi.org/10. 1016/j.ydbio.2014.06.006

Miyazono K, Kamiya Y, Morikawa M (2010) Bone morphogenetic protein receptors and signal transduction. J Biochem 147(1):35-51. https://doi.org/10.1093/jb/mvp148

Nie X, Luukko K, Kettunen P (2006) BMP signalling in craniofacial development. Int J Dev Biol 50(6):511-521. https://doi.org/10. 1387/ijdb.052101xn

Norrie JL, Lewandowski JP, Bouldin CM, Amarnath S, Li Q, Vokes MS, Ehrlich LI, Harfe BD, Vokes SA (2014) Dynamics of BMP signaling in limb bud mesenchyme and polydactyly. Dev Biol 393(2): 270-281. https://doi.org/10.1016/j.ydbio.2014.07.003

Okada H, Gosain AK (2012) Current approaches to management of nonsyndromic craniosynostosis. Curr Opin Otolaryngol Head Neck Surg 20(4):310-317. https://doi.org/10.1097/MOO.0b013e328355a869

Olshan AF, Faustman EM (1989) Nitrosatable drug exposure during pregnancy and adverse pregnancy outcome. Int J Epidemiol 18(4): 891-899

Rahman MS, Akhtar N, Jamil HM, Banik RS, Asaduzzaman SM (2015) TGF-beta/BMP signaling and other molecular events: regulation of osteoblastogenesis and bone formation. Bone Res 3:15005. https:// doi.org/10.1038/boneres.2015.5

Rhinn M, Dolle P (2012) Retinoic acid signalling during development. Development 139(5):843-858. https://doi.org/10.1242/dev.065938

Rice DP (2008) Developmental anatomy of craniofacial sutures. Front Oral Biol 12:1-21. https://doi.org/10.1159/0000115028

Rosen V (2006) BMP and BMP inhibitors in bone. Ann N Y Acad Sci 1068:19-25. https://doi.org/10.1196/annals.1346.005

Rottgers SA, Gallo P, Gilbert J, Macisaac Z, Cray J, Smith DM, Mooney MP, Losee J, Kathju S, Cooper G (2016) Application of laser capture microdissection to craniofacial biology: characterization of anatomically relevant gene expression in normal and craniosynostotic rabbit sutures. Cleft Palate Craniofac J. https://doi.org/10.1597/15-114

Sahar DE, Longaker MT, Quarto N (2005) Sox9 neural crest determinant gene controls patterning and closure of the posterior frontal cranial suture. Dev Biol 280(2):344-361. https://doi.org/10.1016/j.ydbio. 2005.01.022

Saito H, Yamamura K, Suzuki N (2012) Reduced bone morphogenetic protein receptor type 1A signaling in neural-crest-derived cells causes facial dysmorphism. Dis Model Mech 5(6):948-955. https://doi.org/10.1242/dmm.009274

Sanchez-Lara PA, Carmichael SL, Graham JM Jr, Lammer EJ, Shaw GM, Ma C, Rasmussen SA (2010) Fetal constraint as a potential risk factor for craniosynostosis. Am J Med Genet A 152A(2):394 400. https://doi.org/10.1002/ajmg.a.33246

Shen K, Krakora SM, Cunningham M, Singh M, Wang X, Hu FZ, Post JC, Ehrlich GD (2009) Medical treatment of craniosynostosis: recombinant Noggin inhibits coronal suture closure in the rat craniosynostosis model. Orthod Craniofacial Res 12(3):254-262. https:// doi.org/10.1111/j.1601-6343.2009.01460.x
Sieber C, Kopf J, Hiepen C, Knaus P (2009) Recent advances in BMP receptor signaling. Cytokine Growth Factor Rev 20(5-6):343-355. https://doi.org/10.1016/j.cytogfr.2009.10.007

Tsukamoto S, Mizuta T, Fujimoto M, Ohte S, Osawa K, Miyamoto A, Yoneyama K, Murata E, Machiya A, Jimi E, Kokabu S, Katagiri T (2014) Smad9 is a new type of transcriptional regulator in bone morphogenetic protein signaling. Sci Rep 4:7596. https://doi.org/ 10.1038/srep07596

Warren SM, Brunet LJ, Harland RM, Economides AN, Longaker MT (2003) The BMP antagonist noggin regulates cranial suture fusion. Nature 422(6932):625-629. https://doi.org/10.1038/nature01545

Whitton A, Hyzy SL, Britt C, Williams JK, Boyan BD, OlivaresNavarrete R (2016) Differential spatial regulation of BMP molecules is associated with single-suture craniosynostosis. J Neurosurg Pediatr 18(1):83-91. https://doi.org/10.3171/2015.12. PEDS15414

Wilkie AO, Byren JC, Hurst JA, Jayamohan J, Johnson D, Knight SJ, Lester T, Richards PG, Twigg SR, Wall SA (2010) Prevalence and complications of single-gene and chromosomal disorders in craniosynostosis. Pediatrics 126(2):e391-e400. https://doi.org/10.1542/ peds.2009-3491

Wilkie AO, Morriss-Kay GM (2001) Genetics of craniofacial development and malformation. Nat Rev Genet 2(6):458-468. https://doi. org/10.1038/35076601

Wu M, Chen G, Li YP (2016) TGF-beta and BMP signaling in osteoblast, skeletal development, and bone formation, homeostasis and disease. Bone Res 4:16009. https://doi.org/10.1038/boneres.2016.9

Xu Y, Malladi P, Chiou M, Longaker MT (2007) Isolation and characterization of posterofrontal/sagittal suture mesenchymal cells in vitro. Plast Reconstr Surg 119(3):819-829. https://doi.org/10.1097/01.prs. 0000255540.91987.a0

Yamamoto Y, Oelgeschlager M (2004) Regulation of bone morphogenetic proteins in early embryonic development. Naturwissenschaften 91(11):519-534. https://doi.org/10.1007/s00114-004-0575-z

Yang C, Yang L, Wan M, Cao X (2010) Generation of a mouse model with expression of bone morphogenetic protein type II receptor lacking the cytoplasmic domain in osteoblasts. Ann N Y Acad Sci 1192:286-291. https://doi.org/10.1111/j.1749-6632.2009.05248.x

Yip JE, Kokich VG, Shepard TH (1980) The effect of high doses of retinoic acid on prenatal craniofacial development in Macaca nemestrina. Teratology 21(1):29-38. https://doi.org/10.1002/tera. 1420210105

Zeiger JS, Beaty TH, Hetmanski JB, Wang H, Scott AF, Kasch L, Raymond G, Jabs EW, VanderKolk C (2002) Genetic and environmental risk factors for sagittal craniosynostosis. J Craniofac Surg 13(5):602-606

Zhao M, Harris SE, Horn D, Geng Z, Nishimura R, Mundy GR, Chen D (2002) Bone morphogenetic protein receptor signaling is necessary for normal murine postnatal bone formation. J Cell Biol 157(6): $1049-1060$ 